\title{
A Case of Autosomal Recessive Interferon Alpha/Beta Receptor Alpha Chain (IFNAR1) Deficiency with Severe COVID-19
}

\author{
Shaghayegh Khanmohammadi ${ }^{1,2} \cdot$ Nima Rezaei $^{1,2,3} \cdot$ Mehdi Khazaei $^{4} \cdot$ Afshin Shirkani $^{5}$ (i)
}

Received: 19 August 2021 / Accepted: 23 October 2021 / Published online: 28 October 2021

(c) The Author(s), under exclusive licence to Springer Science+Business Media, LLC, part of Springer Nature 2021

\begin{abstract}
Background Interferons (IFNs) play a crucial role in antiviral immunity. Genetic defects in interferon receptors, IFNs, and auto-antibodies against IFNs can lead to the development of life-threatening forms of infectious diseases like a severe form of COVID-19.

Case Presentation A 13-year-old boy with a previously reported homozygous loss-of-function mutation in interferon alpha/ beta receptor subunit 1 (IFNAR1) (c.674-2A > G) was diagnosed with severe COVID-19. He had cold symptoms and a high-grade fever at the time of admission. He was admitted to the pediatric intensive care unit after showing no response to favipiravir and being hypoxemic. High-resolution computed tomography (HRCT) scanning revealed lung involvement of $70 \%$ with extensive areas of consolidation in both lungs. Antibiotics, interferon gamma (IFN- $\gamma$ ), remdesivir, methylprednisolone pulse, and other medications were started in the patient. However, remdesivir and methylprednisolone pulse were discontinued because of their adverse side effects in the patient. His general condition improved, and a few days later was discharged from the hospital.

Conclusion We reported a patient with severe COVID-19 who had a mutation in IFNAR1. Our finding suggests that patients with IFNAR1 deficiency are prone to severe forms of COVID-19. Besides, IFN- $\gamma$ therapy may be a potential drug to treat patients with defects in IFN- $\alpha / \beta$ signaling pathways which needs further investigations.
\end{abstract}

Keywords COVID-19 $\cdot$ SARS-CoV-2 $\cdot$ Coronavirus $\cdot$ IFNAR1 deficiency $\cdot$ Interferon-gamma $\cdot$ IFNs

Afshin Shirkan

a.shirkani@bpums.ac.ir

1 Research Center for Immunodeficiencies, Pediatrics Center of Excellence, Children's Medical Center, Tehran University of Medical Sciences, Tehran, Iran

2 Network of Immunity in Infection, Malignancy and Autoimmunity (NIIMA), Universal Scientific Education and Research Network (USERN), Tehran, Iran

3 Department of Immunology, School of Medicine, Tehran University of Medical Sciences, Tehran, Iran

4 Radiology Department, School of Medicine, Bushehr University of Medical Science, Bushehr, Iran

5 Allergy and Clinical Immunology Department, School of Medicine, Bushehr University of Medical Science, Moallem St., Bushehr, Iran

\section{Introduction}

Interferon alpha/beta receptor subunit 1 (IFNAR1), located on chromosome 21q22.11, encodes a type I membrane protein that, together with IFNAR2, compose interferon alpha/ beta receptor (IFNAR) [1]. Generally, IFNAR2 has a higher affinity for ligands, but recent studies have shown that IFN- $\beta$ has the tightest binding among IFNs, which is associated with IFNAR1 [2]. Activation of IFNAR by IFN- $\alpha$ and IFN- $\beta$ leads to tyrosine phosphorylation of several proteins, such as Janus kinase 1 (JAK1), tyrosine kinase 2 (TYK2), signal transducer and activator of transcription (STAT) proteins, and the subunits of IFNAR themselves in JAK-STAT signaling pathway [1]. IFNAR1 interacts with TYK2, and IFNAR2 interacts with JAK1 and STAT proteins. Then, STAT proteins become transported to the nucleus and act as transcription factors inducing/suppressing the expression of interferon-stimulated genes (ISGs) that are associated with the immune system [2]. STAT proteins play their aforementioned role by interferon 
regulatory factor 9 (IRF9) to form the ISG factor 3 (ISGF3) complex [3].

Type I interferons are mainly known for their antiviral activity. They play their role by several mechanisms, such as inhibiting viral replication in the early stages of infection, upregulating the effector function of immune cells, triggering the adaptive immune response, and increasing antigen presentation [4]. The production of type I interferons is triggered by pathogen recognition receptors, such as toll-like receptors (TLRs), retinoic acid-inducible gene I (RIG-I)-like receptors (RLRs), or cGAS-STING pathway [5-7]. Individuals with mutations in interferon receptors or signaling pathways, and insufficient interferon production show an inadequate antiviral response to the viral infections, which leads to the development of the life-threatening form of the disease [8]. There are several reports of adverse reactions to live attenuated vaccines, such as measles, mumps, and rubella (MMR), and yellow fever vaccine in patients with IFNAR1 or IFNAR2 deficiency or auto-antibodies against type I IFNs [8-11]. Moreover, severe viral infections like herpes simplex encephalitis were seen in the IFNAR1 deficient patients [12].

Investigations in individuals with coronavirus disease (COVID-19) showed that patients with genetic defects in the TLR pathway or IFNAR are more prone to developing severe forms of COVID-19-associated pneumonia than others $[5,13]$. Auto-antibodies against type I IFNs, such as IFN- $\alpha 2$ and IFN- $\omega$, are another common risk factor for developing severe COVID-19 that mimics inborn errors of immunity [14, 15]. Previously, type I interferons were administered to treat COVID-19 patients with a genetic defect in the production of type I interferon pathways, which improved their symptoms [16]. The result of a clinical trial showed that administration of IFN- $\beta$ decreases the time to clinical improvement in COVID19 patients [17]. Another study revealed the positive effect of interferon-gamma (IFN- $\gamma$ ) (type II interferon) in patients with moderate COVID-19 [18]. Moreover, low levels of IFN- $\gamma$ were related to developing lung fibrosis after COVID-19, in a study by Hu et al. [19]. Hence, type I and II IFNs may be a potential drug for treating COVID-19 patients with inborn type I interferon immunity errors. However, IFNAR deficiency complicates the administration of IFN- $\alpha / \beta$ for patients with COVID-19 since they have impaired receptors for type I interferons. Considering this fact, we reported a case of homozygous IFNAR1 deficiency infected with SARS-CoV-2 that developed the severe form of COVID-19 and was treated with IFN- $\gamma$.

\section{Case Presentation}

A 13-year-old boy with IFNAR1 deficiency [9] was admitted to Bushehr Khalij Fars Hospital, Bushehr, Iran, in April 2021. He presented with upper respiratory tracts infection symptoms, such as runny nose, itchy throat, and low-grade fever for 11 days, followed by sweating and high-grade fever in the last 3 days. The patient had a history of meningitis after getting vaccinated for MMR at 12 months of age. He also had a history of recurrent hospitalizations (three hospitalizations in the last 4 years) due to infection with the influenza virus and was treated with IFN- $\gamma$ and oseltamivir. He was a suspected case of COVID-19, confirmed by a positive COVID-19 polymerase chain reaction (PCR) test.

The treatment was initiated with favipiravir; however, the patient showed no response to the therapy, and his symptoms worsened, including shortness of breath and persistent fevers. Therefore, he was admitted to the pediatric intensive care unit (PICU) 1 day after admission to the hospital. The result of high-resolution computed tomography (HRCT) scanning revealed lung involvement of $70 \%$ with extensive areas of consolidation in both lungs and patchy consolidation in the base of the lungs (see Fig. 1). The result of his echo test was normal. The oxygen saturation $(\mathrm{SpO} 2)$ of the patient at the first emergency department visit was $90 \%$ but decreased to $86-88 \%$ within the first days after hospitalization. Due to the low $\mathrm{SpO} 2$, nasal cannula oxygen therapy was started (with a flow of $8 \mathrm{~L} / \mathrm{min}$ ), but it was not helpful. After hospitalization, the patient received vancomycin, piperacillin, tazobactam, aspirin, famotidine, zinc, vitamin $\mathrm{C}$, remdesivir, and methylprednisolone pulse $(30 \mathrm{mg} / \mathrm{kg})$. Two days after hospitalization, the patient's fever persisted, and the antibiotics were switched to colistin and teicoplanin, and caspofungin was added to his medications. Although the level of IL-6 was high in the patient's serum, tocilizumab was not initiated for the patient due to the prolonged prothrombin time (PT) and partial thromboplastin time (PTT) (see Table 1). The prolonged PT and PTT of the patient could be due to COVID-19.

On the third day of hospitalization, the patient had bradycardia and hypertension; however, the second echo test and cardiology consultation were normal. Atropine was prescribed for the patient to be used in case the heart rate falls below 40 beats/min. The eye examination showed papilledema, and pseudotumor cerebri was suspected. Therefore, remdesivir and methylprednisolone pulse were discontinued (only two doses were administered) since the aforementioned side effects were thought to be related to remdesivir and methylprednisolone pulse. The patient received IFN- $\gamma\left(50 \mu \mathrm{g} / \mathrm{m}^{2}\right)$ between the fourth and seventh days of hospitalization (4 doses). IFN- $\gamma$ was discontinued after the seventh day of hospitalization since it can suscept the patient to develop "cytokine storm." He also received one dose $(1 \mathrm{~g} / \mathrm{kg})$ of intravenous immune globulin (IVIG) on the seventh day of hospitalization. After administration of IFN- $\gamma$, the general condition and $\mathrm{SpO} 2$ of the patient improved (1-2\% per day) from the next day. After the fifth day of hospitalization, his fever was reduced. The $\mathrm{SpO} 2$ 
Fig. 1 Lung HRCTs on admission day (left) and a few days later during hospitalization (right); A Axial HRCT, aortic arch level. Extensive consolidation in upper lobes is seen; $\mathbf{B}$ Axial HRCT, subcarinal level. is turning to ground glass attenuation on the second study; $\mathbf{C}$ Axial HRCT, basilar area. There are a few nodular consolidations in the first study (arrows). Patchy confluent consolidations and ground glass opacities are noted on the second study Patchy confluent consolidation

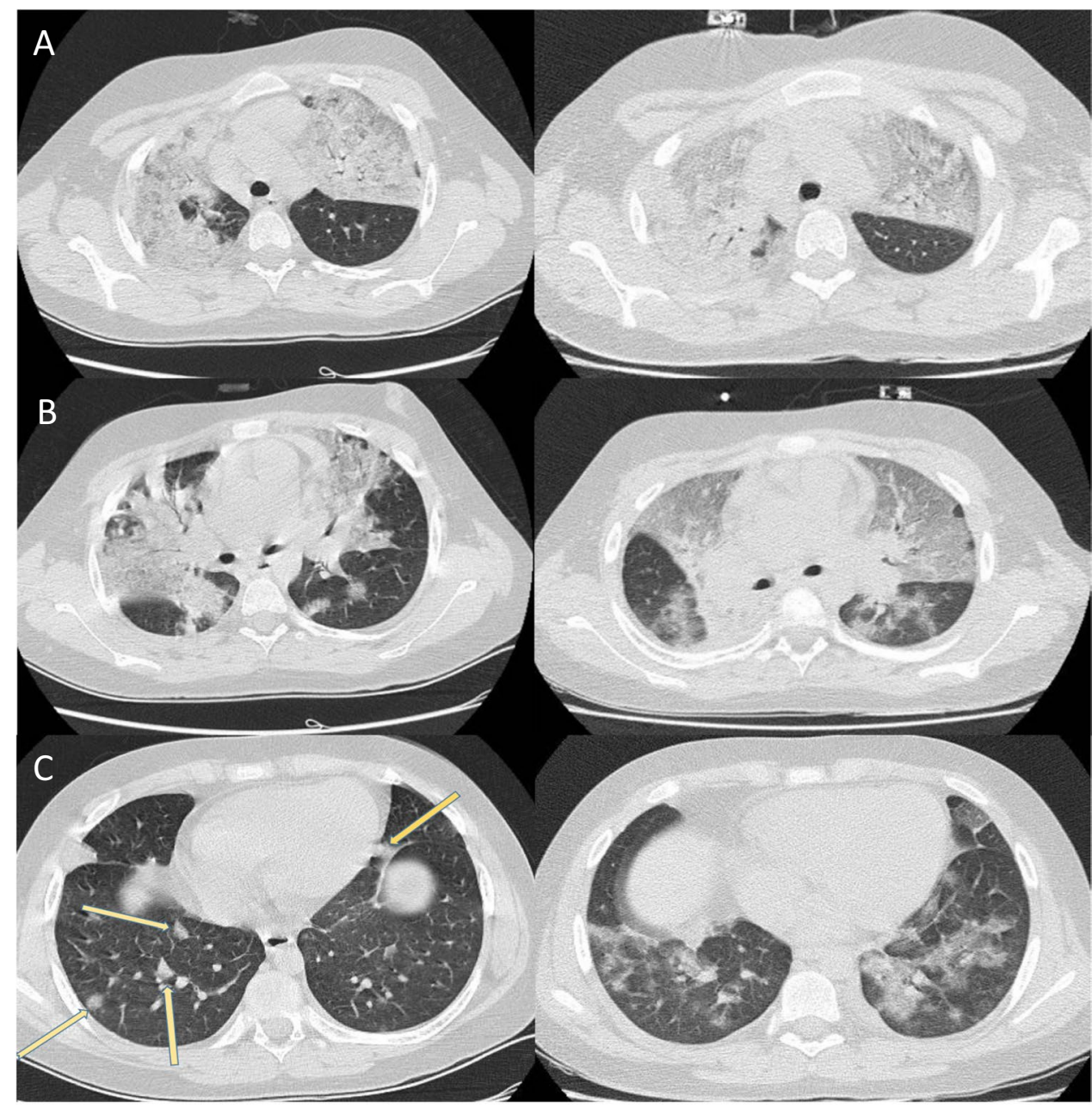

Table 1 Laboratory results

\begin{tabular}{llllll}
\hline Test & Result & Ref. range/unit & Test & Result & Ref. range \\
\hline WBC & $13.2 \rightarrow 14.2 \rightarrow 9.9$ & $4.8-10.810^{\wedge} 3 / \mu \mathrm{L}$ & K & 4.2 & $3.5-5.5 \mathrm{mEq} / \mathrm{L}$ \\
$\mathrm{RBC}$ & 4.5 & $4.5-6.510^{\wedge} 6 / \mu \mathrm{L}$ & BUN & 19 & $6-23 \mathrm{mg} \%$ \\
$\mathrm{Hb}$ & 12 & $13.5-18 \mathrm{~g} / \mathrm{dL}$ & Creatinine & 0.9 & $0.7-1.4 \mathrm{mg} \%$ \\
$\mathrm{PLT}$ & 244 & $150-45010^{\wedge} 3 / \mu \mathrm{L}$ & Calcium & 9.3 & $8.6-10.3 \mathrm{mg} / \mathrm{dL}$ \\
$\mathrm{ESR}$ & $70 \rightarrow 89 \rightarrow 70$ & $0-15 \mathrm{~m} \mathrm{~m} / \mathrm{h}$ & Phosphorus & 3.1 & $3.2-5.7 \mathrm{mg} / \mathrm{dL}$ \\
$\mathrm{IL}-6$ & 36 & $<6.6 \mathrm{pg} / \mathrm{mL}$ & ALT & 49 & $5-40 \mathrm{IU} / \mathrm{L}$ \\
$\mathrm{PT}$ & $14.6 \rightarrow 17.2 \rightarrow 16.1$ & $11-14 \mathrm{~s}$ & ALP & 400 & $35-130 \mathrm{IU} / \mathrm{L}$ \\
$\mathrm{PTT}$ & $47.7 \rightarrow 38.1 \rightarrow 38.1$ & $25-40 \mathrm{~s}$ & Bilirubin total & 0.5 & $0.3-1.2 \mathrm{mg} / \mathrm{dL}$ \\
$\mathrm{INR}$ & $1.36 \rightarrow 1.24 \rightarrow 1.16$ & $0.8-1.2$ & Bilirubin direct & 0.1 & $<0.3 \mathrm{mg} / \mathrm{dL}$ \\
$\mathrm{Na}$ & 136 & $136-145 \mathrm{mEq} / \mathrm{L}$ & CPK & 53 & $10-120 \mu \mathrm{g} / \mathrm{L}$ \\
\hline
\end{tabular}

$W B C$, white blood cell; $R B C$, red blood cell; $H b$, hemoglobin; $P L T$, platelet; $E S R$, erythrocyte sedimentation rate; $P T$, prothrombin time; $P T T$, partial thromboplastin time; $I N R$, international normalized ratio; $I L$ 6, interleukin-6; $B U N$, blood urea nitrogen; $A L T$, alanine transaminase; $A L P$, alkaline phosphatase; $C P K$, creatine kinase of the patient increased to $94 \%$ with nasal cannula oxygen therapy (with a flow of $6 \mathrm{~L} / \mathrm{min}$ ). The antibiotic therapy was continued for 10 days, and the patient received fresh frozen plasma (FFP) $(10 \mathrm{cc} / \mathrm{kg})$ to treat his prolonged PT and abnormal international normalized ratio (INR).
Eight days after hospitalization, the patient had a good general condition and was moved to the ward from PICU. On the ninth day of hospitalization, the patient's $\mathrm{SpO} 2$ was above $94 \%$ without oxygen therapy, and 2 days later, he was discharged from the hospital. 


\section{IFNAR1 Deficiency}

The presented case was born to consanguineous parents and had a sister who passed away after getting vaccinated for MMR. As mentioned before, the patient had a history of meningitis after getting vaccinated for MMR at 12 months of age that led to his recurrent hospitalization due to fever twice a year. The periodic neutropenia disorder was suspected in the patient. Therefore, to find the underlying genetic cause of the disease, whole-exome sequencing (WES) was performed on this patient as previously described by Hernandez et al. [9]. The result of WES (see Table 2) revealed a novel homozygous mutation in the IFNARI gene at the splicing site of exon 6 (NM_000629 Exon6:c.674(-2b)A > G) which was previously reported by Hernandez et al. [9].

\section{Discussion}

This case report presented a patient with severe COVID-19 who had IFNAR1 deficiency. COVID-19, caused by SARS$\mathrm{CoV}-2$, has been a global concern since its emergence in 2020 [20]. Patients with underlying medical conditions are at increased risk for severe manifestations of SARS-CoV-2. Individuals with impaired IFN response seem to develop a life-threatening form of COVID-19 and other viral infections $[13,21]$. As mentioned before, several reports of adverse reactions to the live attenuated vaccines and severe viral infections in patients with auto-antibodies against type I IFNs or IFNAR $1 / 2$ deficiency indicate the importance of the crucial anti-viral role of type I IFNs in body [11, 14]. Investigations in COVID-19 patients with IFNAR1 deficiency showed that they have impaired response to IFN- $\alpha$ and IFN- $\beta$ [13]. Thus, type I interferon administration in these patients may not have an effective outcome, and other treatment strategies should be considered for them.

We treated our IFNAR1-deficient patient, who was infected with SARS-CoV-2, with IFN- $\gamma$ administration. Although other drugs were also used to treat the patient (remdesivir and methylprednisolone), we think that the significant improvement of the general condition of the patient was due to IFN- $\gamma$. Because the patient developed several side effects that were attributed to remdesivir and methylprednisolone, we discontinued them. Additionally, the $\mathrm{SpO} 2$ of the patient increased continuously after the administration of IFN- $\gamma$. Nevertheless, this idea needs further investigations.

IFN- $\gamma$ is a type II interferon produced by activated T cells and natural killer cells that binds interferon-gamma receptor (IFNGR) complex protein, and consequently, activates JAK/STAT pathway [22]. The activation of the JAK/STAT pathway leads to the induction/suppression of the transcription of IFN- $\gamma$-regulated genes [23]. IFN- $\gamma$ has several functions, including antiviral immunity, macrophage activation, $\mathrm{T}$ helper 1 (Th1)/Th2 balance, regulation of apoptosis, and increasing antigen presentation via major histocompatibility complex (MHC) I and MHC II pathway [22]. IFN- $\gamma$ induces antiviral immunity via inhibiting viral entry, suppressing virus replication, inducing cytokine production by immune cells, and enhancing the cytotoxic T lymphocyte killing activity and phagocytosis. Nevertheless, due to the pro-inflammatory nature of the IFN- $\gamma$, persistently high levels of IFN- $\gamma$ can lead to the occurrence of "cytokine storm" [24]. A study by Gadotti et al. revealed that higher levels of IFN- $\gamma$ could cause death in COVID-19 patients [24]. Therefore, IFN- $\gamma$ is a double-edged sword in the management of COVID-19.

The pathway of type I interferon (IFN- $\alpha / \beta)$ and type II interferon (IFN- $\gamma$ ) substantially overlaps. Apparently, IFN$\alpha / \beta$ and IFN- $\gamma$ can cross-talk augment each other's functions [23]. Administration of type I IFN in patients with IFNAR1 deficiency is ineffective. Based on this fact, we considered IFN- $\gamma$ therapy for our patient. It should be noted that we discontinued IFN- $\gamma$ therapy after the seventh day of hospitalization since the persistently high levels of IFN- $\gamma$ may lead to "cytokine storm" in the later stages of the disease [25].

As mentioned before, on the third day of hospitalization, our patient showed symptoms similar to Cushing's reflex [26], which indicates increased intracranial pressure. The corticosteroid therapy was discontinued in the patient since pseudotumor cerebri is one of the side effects of

Table 2 Exome sequencing result

\begin{tabular}{|c|c|c|c|c|c|c|c|c|}
\hline Gene & DNA change & Protein change & dbSNP rsID ${ }^{\mathrm{a}}$ & Associated disease & OMIM & Inheritance & Zygosity & $\begin{array}{l}\text { Classification } \\
{\text { (ClinVar })^{\mathrm{b}}}^{\text {Cli }}\end{array}$ \\
\hline IFNARI & $\begin{array}{l}\text { NM_000629 } \\
\text { Exon6:c.674(-2b)A > G }\end{array}$ & Splice site & - & - & 107,450 & $\mathrm{AR}$ & $\begin{array}{l}\text { K:Hom } \\
\text { M:Het } \\
\text { F:Het }\end{array}$ & NR \\
\hline
\end{tabular}

$K$, kid; $F$, father; $M$, mother; Hom, homozygous; Het, heterozygous; $A D$, autosomal dominant; $N R$, not reported; IFNARl, interferon alpha/beta receptor subunit 1

${ }^{a}$ All variants with dbSNPrsID numbers have minor allele frequencies less than $0.5 \%$ unless otherwise stated

${ }^{\mathrm{b}}$ VUS, variant of uncertain significance 
corticosteroids [27]. Unfortunately, due to the patient's critical condition, further investigations for measuring intracranial pressure were not possible. Additionally, remdesivir was also discontinued because of its adverse cardiac effects, including bradycardia [28].

To the best of our knowledge, we reported one of the first cases of severe COVID-19 with IFNAR1 deficiency that is treated with IFN- $\gamma$. Our findings and approach to managing this COVID-19 patient suggest that IFN- $\gamma$ therapy may be a potential drug to treat patients with inborn errors of type I IFN immunity; however, it needs more investigations to be proved. Since patients with IFNAR1 deficiency or autoantibodies against type I IFNs are more susceptible to the severe form of COVID-19, they should be vaccinated as soon as possible.

\section{Author Contribution Conceptualization: AS}

Formal analysis and investigation: SK, NR

Writing - original draft preparation: SK

Writing — review and editing: NR, AS

Funding acquisition: NR

Imaging studies and patient examination: MK, AS

Supervision: AS

All authors read and approved the final manuscript.

Funding This study was supported by a grant from Tehran University of Medical Sciences (98-3-205-46257).

Data Availability All data of this study are included in this published article.

Code Availability Not applicable

\section{Declarations}

Ethics Approval This study is approved by the ethics committee at the Tehran University of Medical Sciences (Approval ID: IR.TUMS. CHMC.REC.1399.002).

\section{Consent to Participate Not applicable}

Consent for Publication Informed consent was obtained from the patient's father for publication of this case report and any accompanying images.

Conflict of Interest The authors declare no competing interests.

\section{References}

1. UniProt. UniProtKB - P17181 (INAR1_HUMAN). Available from: https://www.uniprot.org/uniprot/P17181. Accessed 6 Aug 2021.

2. Schreiber G. The role of type I interferons in the pathogenesis and treatment of COVID-19. Front Immunol. 2020. 11:595739. https:// doi.org/10.3389/fimmu.2020.595739.

3. McNab F, et al. Type I interferons in infectious disease. Nat Rev Immunol. 2015;15(2):87-103.
4. Murira A, Lamarre A. Type-I interferon responses: from friend to foe in the battle against chronic viral infection. Front Immunol. 2016. 7(609). https://doi.org/10.3389/fimmu.2016.00609.

5. Khanmohammadi S, Rezaei N. Role of Toll-like receptors in the pathogenesis of COVID-19. J Med Virol. 2021;93(5):2735-9.

6. Rehwinkel J, Gack MU. RIG-I-like receptors: their regulation and roles in RNA sensing. Nat Rev Immunol. 2020;20(9):537-51.

7. Li A, et al. Activating cGAS-STING pathway for the optimal effect of cancer immunotherapy. J Hematol Oncol. 2019;12(1):35.

8. Duncan CJA, Randall RE, Hambleton S. Genetic lesions of type I interferon signalling in human antiviral immunity. Trends Genet. 2021;37(1):46-58.

9. Hernandez N, et al. Inherited IFNAR1 deficiency in otherwise healthy patients with adverse reaction to measles and yellow fever live vaccines. J Exp Med. 2019;216(9):2057-70.

10. Gothe F, Hatton CF, Truong L, Klimova Z, Kanderova V, Fejtkova $\mathrm{M}$ et al. A novel case of homozygous IFNAR1 deficiency with haemophagocytic lymphohistiocytosis. Clin Infect Dis. 2020;30. https:// doi.org/10.1093/cid/ciaa1790.

11. Bastard P, Michailidis E, Hoffmann HH, Chbihi M, Le Voyer T, Rosain J et al. Auto-antibodies to type I IFNs can underlie adverse reactions to yellow fever live attenuated vaccine. J Exp Med. 2021;218(4). https://doi.org/10.1084/jem.20202486.

12. Bastard P, Manry J, Chen J, Rosain J, Seeleuthner Y, AbuZaitun O et al. Herpes simplex encephalitis in a patient with a distinctive form of inherited IFNAR1 deficiency. J Clin Invest. 2021;131(1). https:// doi.org/10.1172/JCI139980.

13. Zhang Q, Bastard P, Liu Z, Le Pen J, Moncada-Velez M, Chen J et al. Inborn errors of type I IFN immunity in patients with life-threatening COVID-19. Science 2020;370(6515). https://doi.org/10.1126/ science.abd4570.

14. Bastard $\mathrm{P}$, et al. Autoantibodies against type I IFNs in patients with life-threatening COVID-19. Science. 2020;370(6515):eabd4585.

15. Bastard P, et al. Autoantibodies neutralizing type I IFNs are present in $\sim 4 \%$ of uninfected individuals over 70 years old and account for 20\% of COVID-19 deaths. Sci Immunol. 2021;6(62):eabl4340.

16. Lévy R, et al. IFN- $\alpha 2$ a therapy in two patients with inborn errors of TLR3 and IRF3 infected with SARS-CoV-2. J Clin Immunol. 2021;41(1):26-7.

17. Alavi Darazam I, et al. Role of interferon therapy in severe COVID19: the COVIFERON randomized controlled trial. Sci Rep. 2021;11(1):8059.

18. Myasnikov AL, et al. Interferon gamma in the treatment of patients with moderate COVID-19. Vopr Virusol. 2021;66(1):47-54.

19. Hu ZJ, Xu J, Yin JM, Li L, Hou W, Zhang LL et al. Lower circulating interferon-gamma is a risk factor for lung fibrosis in COVID-19 patients. Front Immunol. 2020;11:585647. https://doi.org/10.3389/ fimmu.2020.585647.

20. Khanmohammadi S, Tabary M, Araghi F, Tavangar SM. SARSCoV-2: an insight into detection tools. Infect Disord Drug Targets. 2021. https://doi.org/10.2174/1871526520666210104151849.

21. Hadjadj J, et al. Impaired type I interferon activity and inflammatory responses in severe COVID-19 patients. Science. 2020;369(6504):718.

22. Tau G, Rothman P. Biologic functions of the IFN-gamma receptors. Allergy. 1999;54(12):1233-51.

23. Schroder K, et al. Interferon- $\gamma$ : an overview of signals, mechanisms and functions. J Leukoc Biol. 2004;75(2):163-89.

24. Gadotti AC, et al. IFN- $\gamma$ is an independent risk factor associated with mortality in patients with moderate and severe COVID-19 infection. Virus Res. 2020;289:198171-198171.

25. Cappanera $\mathrm{S}$, et al. When does the cytokine storm begin in COVID-19 patients? A quick score to recognize it. J Clin Med. 2021;10(2):297. 
26. Dinallo $S$ and Waseem M, Cushing reflex, in StatPearls. 2021, StatPearls Publishing Copyright (c) 2021, StatPearls Publishing LLC.: Treasure Island (FL).

27. Ivey KJ, DenBesten L. Pseudotumor Cerebri associated with corticosteroid therapy in an adult. JAMA. 1969;208(9):1698-700.
28. Touafchia A, et al. Serious bradycardia and remdesivir for coronavirus 2019 (COVID-19): a new safety concerns. Clin Microbiol Infect. 2021;27(5):791.e5-791.e8.

Publisher's Note Springer Nature remains neutral with regard to jurisdictional claims in published maps and institutional affiliations. 